\title{
Calycosin inhibits migration and invasion through modulation of transforming growth factor beta- mediated mesenchymal properties in U87 and U25 I cells
}

\author{
This article was published in the following Dove Press journal: \\ Drug Design, Development and Therapy \\ 22 February 2016 \\ Number of times this article has been viewed
}

Xiao-hu Niel,*
Jia Ou-yang,*
Ying Xing
Dan-yan Li
Ru-en Liu'
Ru-xiang Xu'
'Department of Neurosurgery,
Huzhou Central Hospital, Huzhou,
Zhejiang, ${ }^{2}$ Nanchang University
Medical College, Nanchang, Jiangxi,
${ }^{3}$ Department of Gastroenterology,
The 98 th Hospital of Nanjing
Military Command, Huzhou, Zhejiang,
${ }^{4}$ Spleen \& Stomach Institute,
Guangzhou University of Traditional
Chinese Medicine, Guangzhou,
Guangdong, ${ }^{5}$ Department of
Neurosurgery, China-Japan Friendship
Hospital, Beijing, ${ }^{6}$ Bayi Brain Hospital,
The Military General Hospital of
Beijing PLA, Beijing, People's Republic
of China
*These authors contributed equally
to this work

Correspondence: Ru-en Liu

Department of Neurosurgery,

China-Japan Friendship Hospital,

Yinghua East Street, Beijing 100029,

People's Republic of China

$\mathrm{Tel}+86 \quad 10 \quad$ I339 I8I7 478

$\mathrm{Fax}+861084645296$

Email liure@।26.com

Ru-xiang Xu

Bayi Brain Hospital, The Military

General Hospital of Beijing PLA,

No 5 Nanmen Cang, Beijing I00700,

People's Republic of China

Tel +86 I0 I339 I788 I | 8

Fax +86106405 7752

Email bbhxuruxiang@|26.com

\begin{abstract}
In this study, we investigated the potential anticancer effects of calycosin against human glioblastoma cells, including the impacts on cell proliferation, apoptosis, and cell cycle distribution. We further studied its inhibitory activity on migration and invasion in U87 and U251 cells. Furthermore, transforming growth factor beta-mediated reductions of mesenchymal-associated genes/activators, matrix metalloproteinases- 2 , and -9 were detected in this process. Administration of calycosin in a glioblastoma xenograft model showed that calycosin could not only reduce tumor volume but also suppress transforming growth factor beta as well as its downstream molecules. These results revealed calycosin as a potential antitumor agent in human glioblastoma.
\end{abstract}

Keywords: calycosin, migration, invasion, epithelial-mesenchymal transition (EMT), matrix metalloproteinases (MMPs), glioblastoma

\section{Introduction}

Glioblastomas are the most common primary malignant cancer in the central nervous system. ${ }^{1}$ Surgery combined with chemotherapy remains the standard treatment for glioblastomas. ${ }^{2}$ Despite the development of aggressive treatments, most malignant gliomas are resistant to chemotherapeutic agents and patients have a mean survival of 14.6 months from the time of diagnosis. ${ }^{3}$ Thus, a novel therapeutic approach to cure this malignancy is in urgent need.

Epithelial-mesenchymal transition (EMT) is an important process in tumor genesis, metastasis, and recurrence that allows immobile epithelial cells to undergo biochemical changes and induces a mesenchymal cell phenotype, including enhanced migratory properties, invasiveness, and resistance to apoptosis..$^{4,5}$ Although this concept in the neuroepithelial context is currently controversially discussed, there are plenty of evidences that mesenchymal (like) transition in glioblastoma is associated with poor prognosis and aggressive internal malignancy. ${ }^{6-8}$

The isoflavone calycosin is a main active compound of Radix astragali. Studies indicated that calycosin had strong antioxidant, antiviral, and immunomodulatory effects. ${ }^{9-11}$ Some recent researches also indicated that calycosin might be a potent antitumor reagent. ${ }^{12-14}$ However, the anticancer activity of calycosin on glioma cells has not been investigated yet.

In the present study, we demonstrated the antineoplastic activity of calycosin on glioblastomas in vitro and in vivo. More importantly, we further explored its inhibitory

submit your manuscript | www.dovepress.com 
effects on mesenchymal properties, matrix metalloproteinase (MMP) -2 and MMP-9, as well as its underlying mechanisms.

\section{Materials and methods}

\section{Chemicals, reagents, antibodies, and cell culture}

Calycosin was purchased from Tianjin SHILAN Technology (\#PCM-AM-005, Tianjin, People's Republic of China). Antibodies specific for transforming growth factor beta (TGF- $\beta$ ) (\#3709), N-cadherin (\#13116), Snail (\#3879), Vimentin (\#5741), MMP-2 (\#13132), MMP-9 (\#4022), and $\beta$-actin (\#3700) were obtained from Cell Signaling Technology (Danvers, MA, USA) and used as primary antibodies in the Western blot. Human U87 and U251 cell lines were obtained from the Chinese Academy of Medical Sciences (Beijing, People's Republic of China). Cells were maintained in Dulbecco's Modified Eagle's Medium (\#41966-052, Thermo Fisher Scientific, Waltham, MA, USA) supplemented with 10\% fetal bovine serum (\#10099-141, Invitrogen).

\section{MTT assay}

The viability of cells was determined by 3-(4,5)-dimethylthiahiazo (-z-y1)-3,5-di- phenytetra zoliumromide (MTT) assay. Cells $\left(4 \times 10^{3}\right.$ per well) were seeded in triplicate on a $96-$ well plate and incubated overnight before treatment with calycosin $(0-800 \mu \mathrm{M})$ for 24 hours. The absorbance was measured on an ELISA reader (Multiskan EX; Labsystems; Dynex Technologies, Denkendorf, Germany) at $490 \mathrm{~nm}$.

\section{Annexin V-FITC/PI staining}

In all, $1.5-2 \times 10^{5}$ cells were plated into six-well plates, incubated with calycosin $(0-800 \mu \mathrm{M})$ for 24 hours, and then collected and stained with Annexin V-FITC/propidium iodide (PI) as per the manufacturer's instructions (Dojindo, Kumamoto, Japan). Briefly, the cells were washed twice in cold phosphate-buffered saline (PBS), resuspended in 100 $\mu \mathrm{L}$ binding buffer, and incubated with $5 \mu \mathrm{L}$ Annexin $\mathrm{V}$ and 5 $\mu \mathrm{L}$ PI for 15 minutes at room temperature in the dark before flow cytometric analysis.

\section{Cell cycle analyses}

Cells were exposed to different concentrations of calycosin for 24 hours, collected, washed, and resuspended with cold PBS. These cells were fixed with $500 \mu \mathrm{L}$ of $70 \%$ ethanol overnight at $4^{\circ} \mathrm{C}$, and then washed twice with cold PBS. Finally, cells were stained with $500 \mu \mathrm{L}$ of PI/RNase Staining Buffer reagent (BD, Franklin Lakes, NJ, USA) for 30 minutes at room temperature before analysis using an Accuri C6 flowcytometer system (BD).

\section{Migration and invasion assays}

To assess cell migration in vitro, cells $\left(1.5 \times 10^{5}\right.$ in $500 \mu \mathrm{L}$ Dulbecco's Modified Eagle's Medium [DMEM] supplemented with $1 \%$ fetal bovine serum [FBS]) were seeded in the upper chamber of transwell migration chambers $(8 \mu \mathrm{m}$ BioCoat Control Inserts, Becton Dickinson Labware, Bedford, MA, USA). The lower chamber was filled with 750 $\mu \mathrm{L}$ DMEM supplemented with $20 \%$ FBS and indicated concentrations of calycosin (100 and $200 \mu \mathrm{M}, 1 / 500$ of dimethyl sulfoxide [DMSO] set as the vehicle). After 18 hours, the cells in the lower chamber were fixed by methanol, stained with $0.1 \%$ crystal violet in methanol, and photographed in three independent $10 \times$ fields for each well. Crystal violet was then dissolved with $33 \%$ acetic acid, and absorbance was read at $570 \mathrm{~nm}$. Invasiveness assay was similarly performed with matrigel-coated (BD Biosciences, San Jose, CA, USA) transwells. And the drug incubation time was prolonged to 24 hours. All the experiments were performed in triplicate and repeated three times.

\section{Western blot analyses}

Proteins from cells or tumor tissues were extracted with ProteoJET ${ }^{\mathrm{TM}}$ Mammalian Cell Lysis Reagent (Fermentas, Burlington, ON, Canada) supplemented with protease and phosphatase inhibitors (Fermentas), according to the manufacturer's instructions. Equal amounts of total protein were separated on $8 \%-10 \%$ sodium dodecyl sulfate-polyacrylamide gel electrophoresis (SDS-PAGE), transferred to polyvinylidene difluoride membranes (Merck KGaA, Darmstadt, Germany), and probed with specific primary antibodies. Signal was obtained using Super Signal ECL (Pierce, Rockford, IL, USA).

\section{Cell transfection}

Semiconfluent U87 cultures in $60 \mathrm{~mm}^{2}$ dishes were transfected with pEGFP or pEGFP-TGF- $\beta 1$ expression vector (Bioworld, Beijing, People's Republic of China) with Lipofectamine ${ }^{\mathrm{TM}} 2000$ reagent following the manufacturer's protocol. Twenty-four hours after transfection, cells were incubated with calycosin/vehicle for another 24 hours or seed in transwell filters for further investigation.

\section{RNA preparation and real-time polymerase chain reaction}

Total RNA was isolated using an E.Z.N.A. Total RNA Kit (Omega Bio-Tek, Norcross, GA, USA). The cDNA was synthesized from $1 \mu \mathrm{g}$ of total RNA using a Prime-Script II 1st Strand cDNA Synthesis Kit (Takara, Shiga, Japan). Gene expression was determined by real-time 
polymerase chain reaction (PCR) using a SYBR Premix Ex Taq Kit (Takara) and an ABI Vii7 detection system (Applied Biosystems, Kumamoto, Japan). The sequences of PCR primers used in this study are listed in Table S1.

\section{Xenograft model}

Six-week-old female nude mice, 20-25 g body weight (BALB/c nu/nu; Vital River Laboratories, Beijing, People's Republic of China) were housed into individually ventilated cages, and free accessed to food and drinking water. An amount of $0.5 \times 10^{7} \mathrm{U} 87$ cells in $0.1 \mathrm{~mL}$ PBS was injected into the right hind flank of the mice. Tumor sizes were measured with a caliper and calculated as follows:

$$
\text { Tumor size }\left(\mathrm{mm}^{3}\right)=1 / 2 \times \text { length } \times \text { width }^{2}
$$

When the tumors grew to approximately $200 \mathrm{~mm}^{3}$, the tumor-bearing mice were randomly divided into two groups ( $n=5 /$ group). Calycosin or PBS was injected intravenously into the mice at doses of $7.5 \mathrm{mg} / \mathrm{kg}$ in $0.2 \mathrm{~mL}$ every other day for 22 days. Tumor sizes and body weights were measured two or three times per week as described earlier. At the end of the experiment, the mice were anesthetized by Zoletil 50 (Virbac Animal Health, Carros, France) and sacrificed via $\mathrm{CO}_{2}$ euthanasia. Tumor tissues were snap frozen in liquid nitrogen and stored at $-80^{\circ} \mathrm{C}$. Individual tumor samples were analyzed for the expression levels of proteins. All animal experiments and use of human cancer cell lines were conducted according to the protocols approved by the Institutional Animal Care and Use Committee of Southern Medical University and the Military General Hospital of Beijing PLA, and the study was approved by the Institutional Animal Care and Use Committee of Southern Medical University and Military General Hospital of Beijing PLA (approval number: 2015019).

\section{Statistical analysis}

The data are presented as the mean \pm standard deviation from at least three independent experiments. Differences were analyzed using one-way analysis of variance (ANOVA) (three or more groups) or independent $t$-test (two groups). $P<0.05$ was considered statistically significant.

\section{Results}

\section{Effects of calycosin on cell proliferation, apoptosis, and cell cycle distribution in U87 and U25I cells}

The cytotoxicity of calycosin on human glioblastoma cells was assessed by MTT assay. As shown in Figure 1A, calycosin inhibited cell proliferation only at higher concentrations $(>400 \mu \mathrm{M})$. However, low achievable calycosin concentrations did not exert potent growth inhibitory effects $(P<0.05)$.

Since induction of apoptosis is critical for effective tumor regression, we next measured apoptosis by Annexin V-FITC/PI staining. Data present here confirmed that $400 \mu \mathrm{M}$ calycosin significantly induced apoptosis in U87 cells $(P<0.05)$. However, there was no significant difference in the apoptotic rate even with up to $800 \mu \mathrm{M}$ calycosin in U251 cells (Figure 1B and C).

We also found that treatment with $800 \mu \mathrm{M}$ calycosin for 24 hours in both cell lines induced cell cycle arrest (Figure 1D): percentage of cells in $\mathrm{G} 2 / \mathrm{M}$ phase increased from $24.8 \%$ to $41.9 \%$ (U87) and $21.1 \%$ to $30.6 \%$ (U251), as well as the percentage of cells in G1 phase decreased from $50.6 \%$ to $30.8 \%$ (U87) and $51.2 \%$ to $28.4 \%$ (U251), respectively.

\section{Calycosin inhibited cell migration and invasion}

Next, we employed transwell assays to investigate if migration and invasion of glioblastoma cells were inhibited by calycosin. As shown in Figure 2, much fewer cells were found to infiltrate the membranes with or without matrigel under the treatment of 100 and $200 \mu \mathrm{M}$ calycosin, compared with untreated groups $(P<0.05)$.

\section{Calycosin inhibited mesenchymal properties and MMPs in glioblastoma cells}

Considering that mesenchymal transition is closely associated with migration and invasion of cancer cells, we examined the changes of molecules related to this process after calycosin treatment. After 24 hours incubation with calycosin for the indicated concentrations $(0,100$, and $200 \mu \mathrm{M})$, the expression of mesenchymalrelated molecules was explored. We found that calycosin significantly decreased the expressions of mesenchymalassociated genes/activators, including N-cadherin, Snail, and Vimentin (Figure 3A, $P<0.05$ ) (Figure 3B). These results strongly suggested that calycosin could elicit pronounced inhibitory effects on mesenchymal properties and its related molecules.

Two key MMPs (MMP-2 and MMP-9) were also evaluated following calycosin treatment. Figure $3 \mathrm{C}$ and D clearly presents that calycosin incubation led to reduced levels of MMP-2 and MMP-9 in a dose-dependent manner $(P<0.05)$. 

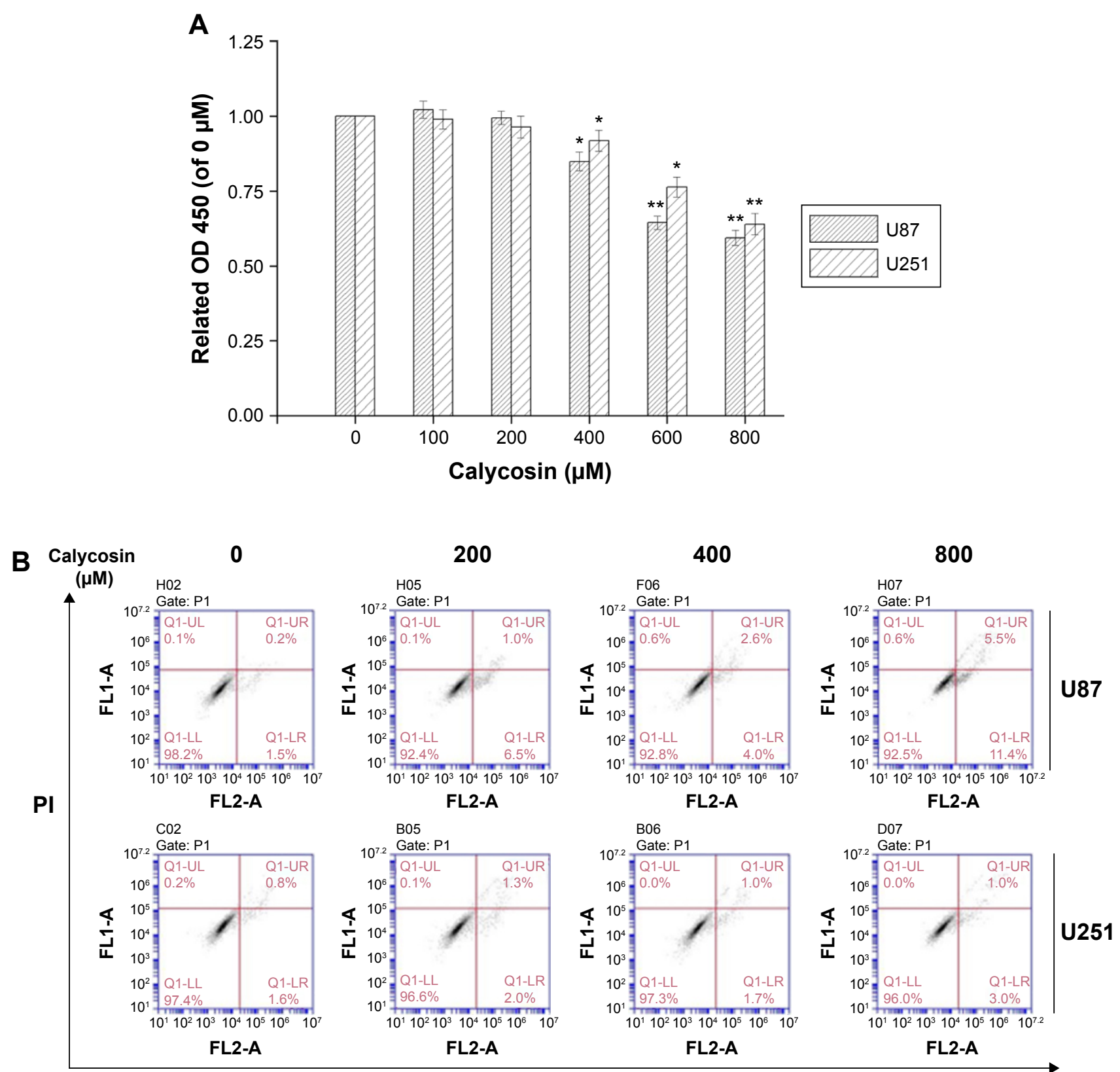

Annexin V-FITC

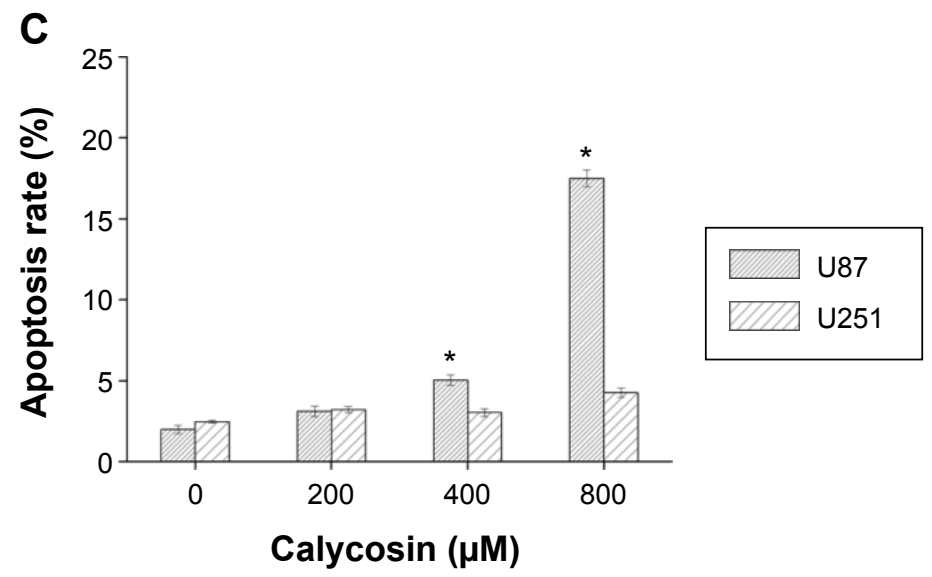

Figure I (Continued) 
D
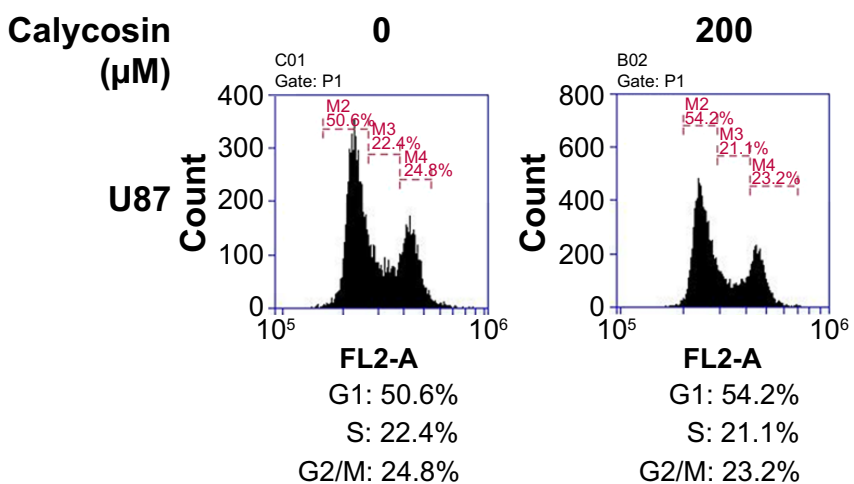

S: $21.1 \%$

G2/M: $23.2 \%$

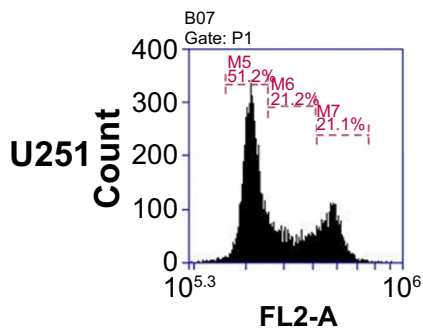

G1: $51.2 \%$

S: $21.2 \%$

G2/M: $21.1 \%$

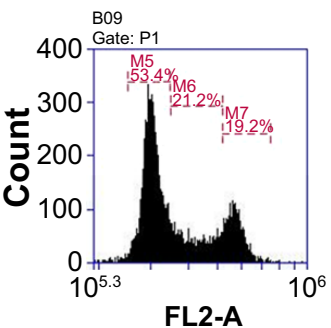

G1: $53.4 \%$

S: $21.2 \%$

G2/M: $19.2 \%$
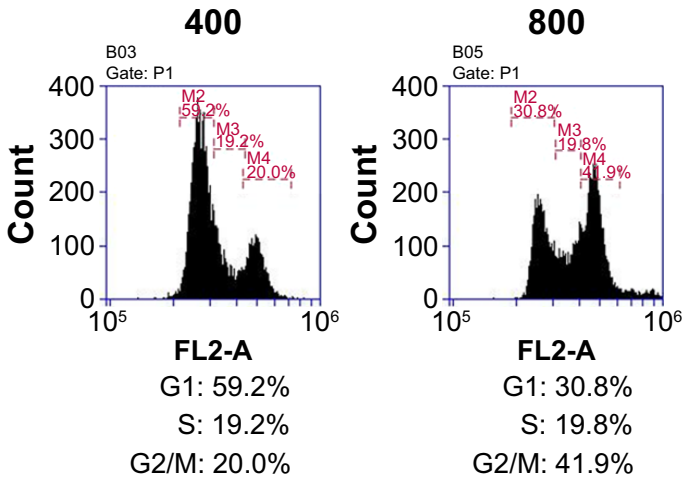

G1: $30.8 \%$

S: $19.8 \%$

G2/M: $41.9 \%$

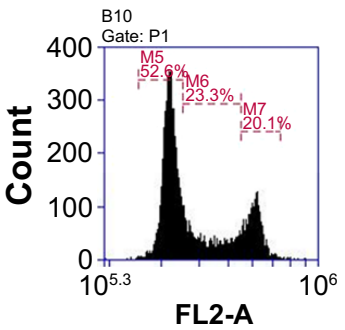

G1: $52.6 \%$

S: $23.3 \%$

G2/M: $20.1 \%$

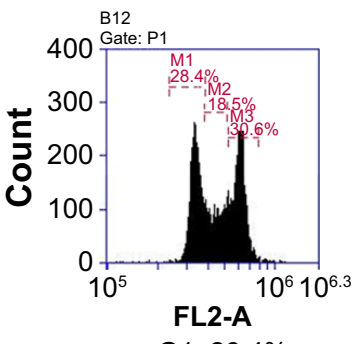

G1: $28.4 \%$

S: $18.5 \%$

G2/M: $30.6 \%$

Figure I Effects of calycosin on proliferation, apoptosis, and cell cycle regulation in U87 and U25I cells.

Notes: (A) Cells were treated with various concentrations of calycosin for 24 hours before MTT assay. (B and C) Cells were treated with the indicated concentrations of calycosin $(0,200,400$, and $800 \mu \mathrm{M})$ for 24 hours and stained with Annexin V-FITC/PI. At least 10,000 cells were tested per sample. All tests were performed in triplicate and presented as mean \pm standard deviation. $* P<0.05$, $* * P<0.0$ l, compared with control $(0 \mu \mathrm{M})$. (D) Cells were exposed to different concentrations of calycosin for 24 hours. After treatment, cells were harvested and subjected to flow cytometric analysis to assess the cell cycle distribution. At least I0,000 cells were analyzed per sample. After treatment, cells were harvested and subjected to flow cytometric analysis to assess the cell cycle distribution. At least I0,000 cells were analyzed per sample. Abbreviations: OD, optical density; PI, propidium iodide.

A

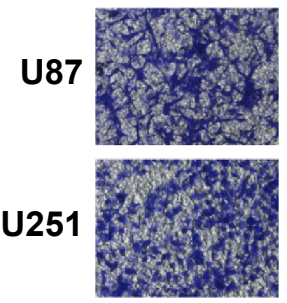

Calycosin $(\mu \mathrm{M})$

C

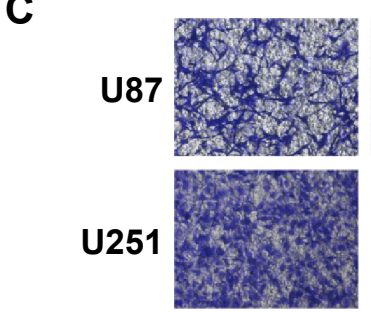

Calycosin $(\mu \mathrm{M})$

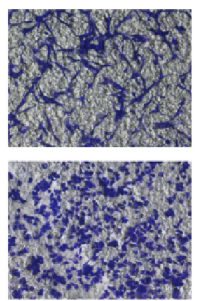

100

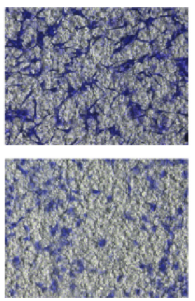

100

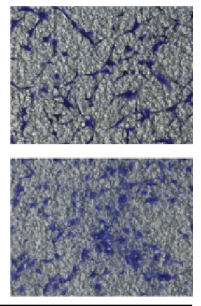

200

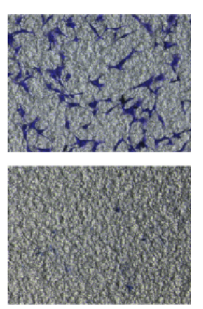

200

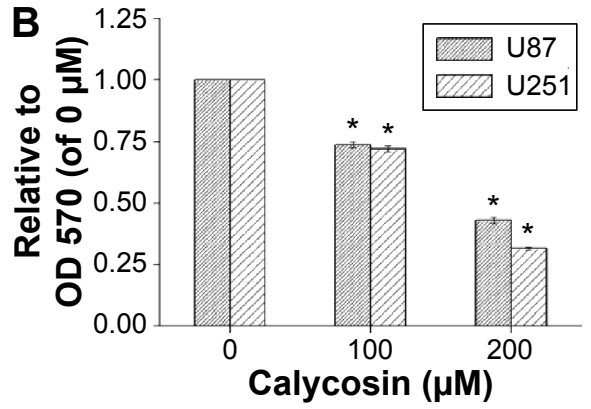

D ${ }^{1.25} \quad$ U87

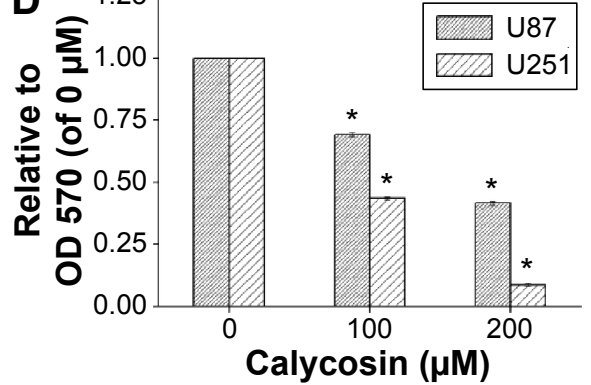

Figure 2 Inhibitory effects of calycosin on cell migration and invasion.

Notes: (A) Cells were treated with indicated concentrations of calycosin for 18 hours. The representative images of migrate cells at the bottom of the membrane stained with crystal violet were visualized. (B) The stained cells were eluted by $33 \%$ glacial acetic acid for 20 minutes. The elution was measured at $570 \mathrm{~nm}$ to obtain the OD 570 values, and normalized to that of control. (C) Cells were incubated with indicated concentrations of calycosin for 24 hours. Photographs are of the cell invasion through the polycarbonate membrane stained by crystal violet. (D) Each treatment had three replicates. All tests were performed in triplicate and presented as mean \pm standard deviation. $* P<0.05$, compared with control $(0 \mu \mathrm{M})$.

Abbreviation: OD, optical density. 
A
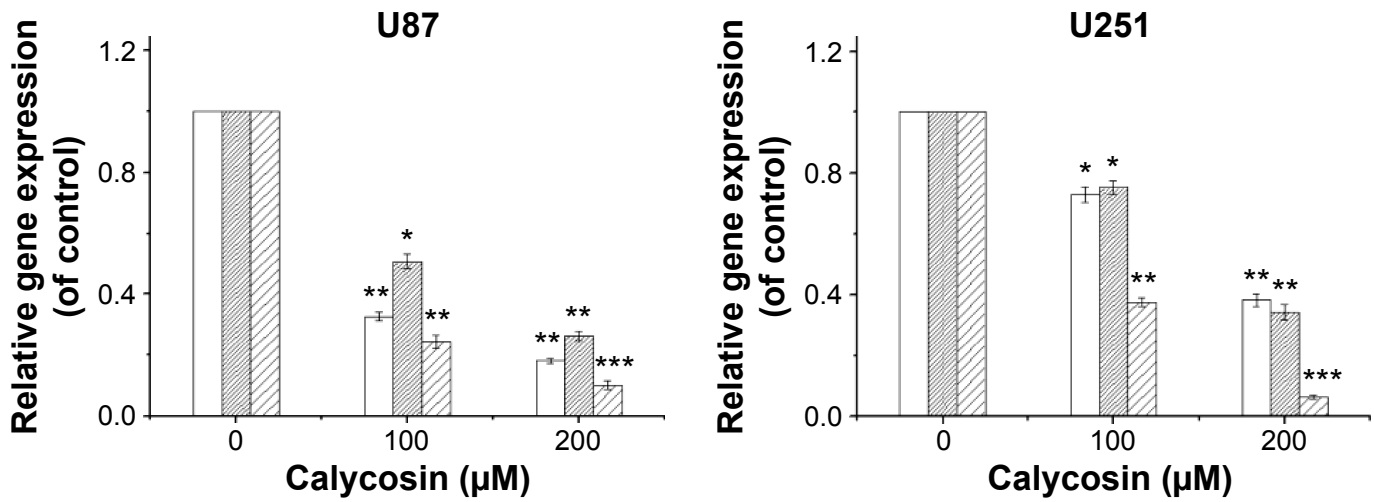

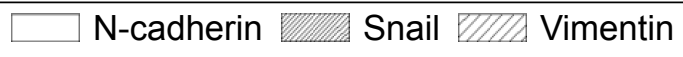

B Calycosin $(\mu \mathrm{M})$

0

$100 \quad 200$

\section{$\mathrm{N}$-cadherin}
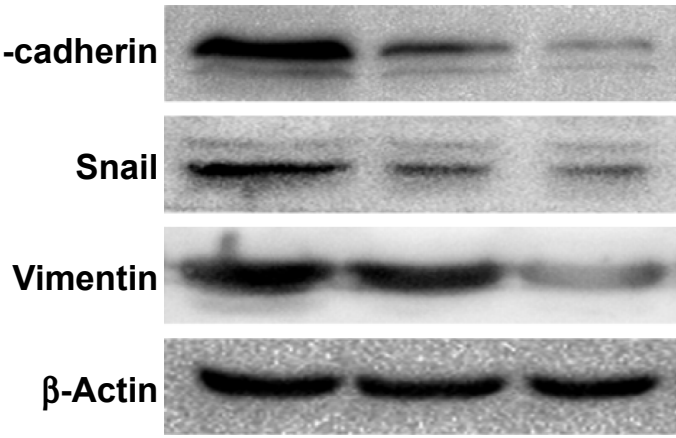

U87
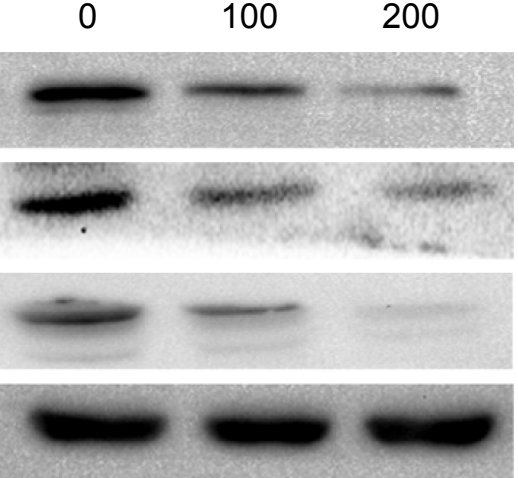

U251

C
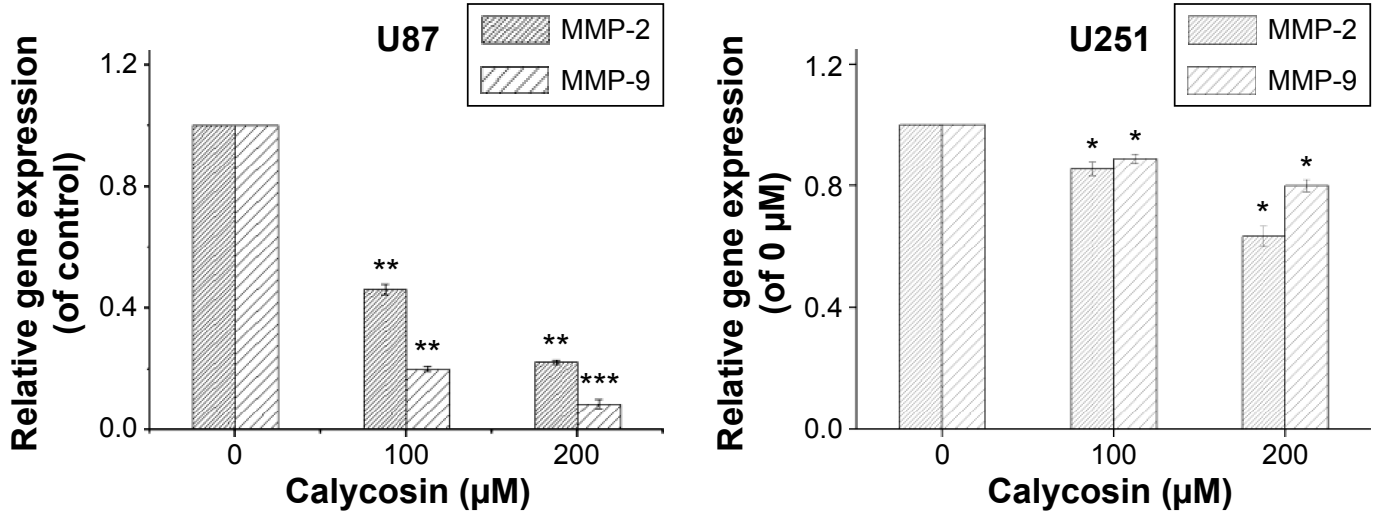

D
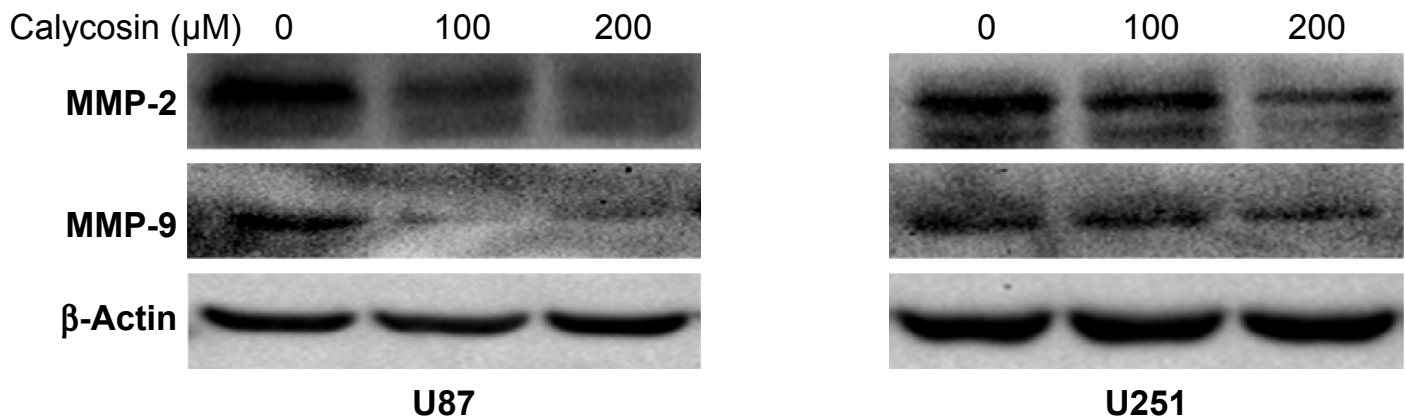

Figure 3 Calycosin inhibited mesenchymal properties and MMPs in U87 and U25I cells.

Notes: (A and B) Dose-response study of calycosin on mesenchymal activator/related genes expression and protein levels in glioblastoma cells. (C and D) Gene expression and protein levels of MMP-2 and MMP-9 in U87 and U25I were evaluated in calycosin treated cells after 24 hours. All tests were performed in triplicate and presented as mean \pm standard deviation. $* P<0.05$, $* * P<0.01$, $* * * P<0.001$, compared with control $(0 \mu \mathrm{M})$.

Abbreviation: MMP, matrix metalloproteinase. 


\section{Calycosin downregulated TGF- $\beta$ in glioblastoma cells}

The ability of calycosin to modulate TGF- $\beta$ in two glioblastoma cell lines was investigated. We found that both mRNA and protein expression were significantly inhibited in a dose-dependent way (Figure 4A and B) $(P<0.05)$.

\section{Ectopic expression of TGF- $\beta$ in U87 cells abrogated the anticancer activity of calycosin}

We then tested whether TGF- $\beta$ overexpression could reverse the antimigration or anti-invasion activity of calycosin. We found that inhibitory effects of calycosin were significantly attenuated in U87 cells which were subjected to forced overexpression of TGF- $\beta 1(P<0.05)$ (Figure $5 \mathrm{~A}-\mathrm{D})$. We also evaluated the effect of ectopic expression of TGF- $\beta 1$ on the expression of mesenchyme-associated molecules and MMPs. As discussed earlier, protein levels of these molecules were significantly downregulated by calycosin (Figure 5E). However, overexpression of TGF- $\beta 1$ restored their levels, suggesting a critical role of TGF- $\beta$ on calycosin-mediated antimesenchymal properties or MMPs' inhibition. Taken together, these findings indicated that TGF- $\beta$ regulate, at least in part, the suppressive effects of calycosin on mesenchymal properties, MMPs' expression, migration, and invasion in glioblastoma.

\section{Effects of calycosin in glioblastoma xenografts}

We employed a U87 xenograft mouse model to further address the anticancer activity of calycosin. Results showed that calycosin statistically reduced tumor volume after calycosin treatment for 22 days compared with the group that received vehicle PBS $(P<0.05)$ (Figure 6A and $B)$. The body weight was monitored until the mice were sacrificed, and no differences in these parameters were observed between the two groups (Figure 6C) $(P>0.05)$. To verify molecular changes in an animal model, the proteins purified from tumor tissues were analyzed using immunoblotting. As demonstrated in Figure 6D, calycosin suppressed TGF- $\beta$ as well as its downstream molecules: N-cadherin, Snail, Vimentin, MMP-2, and MMP-9. These results were in complete agreement with our findings in vitro.

A

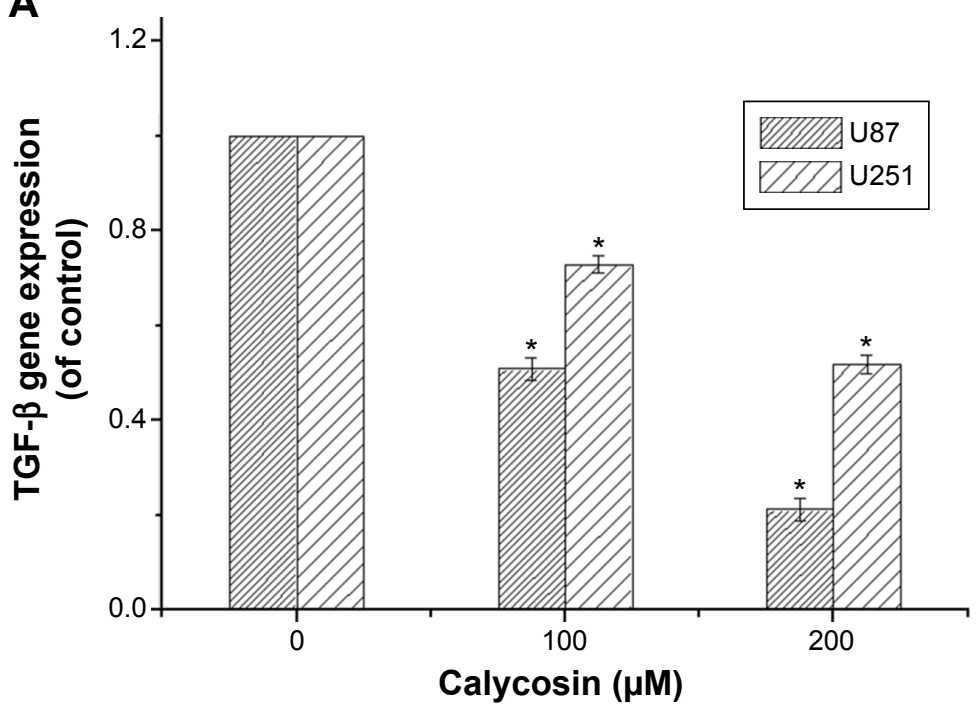

B

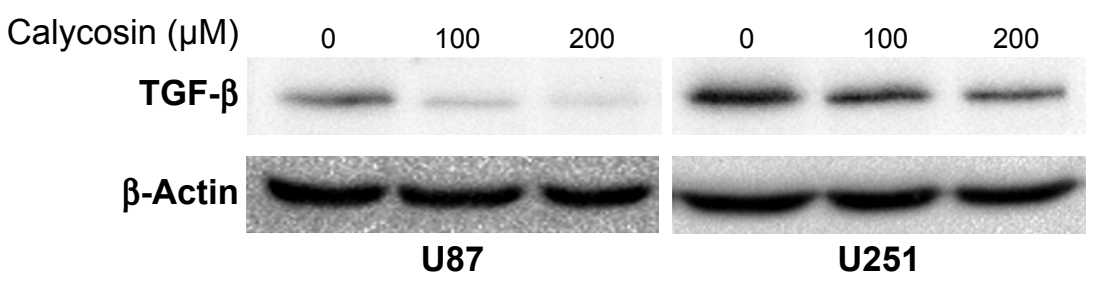

Figure 4 Effects of calycosin on transforming growth factor beta (TGF- $\beta$ ) expression in glioblastoma cells.

Notes: (A and B) Cells were treated with indicated concentrations of calycosin for 24 hours. Gene expression and protein levels of TGF- $\beta$ were evaluated by real-time PCR and Western blot, respectively. $* P<0.05$, compared with control $(0 \mu \mathrm{M})$.

Abbreviation: $\mathrm{PCR}$, polymerase chain reaction. 


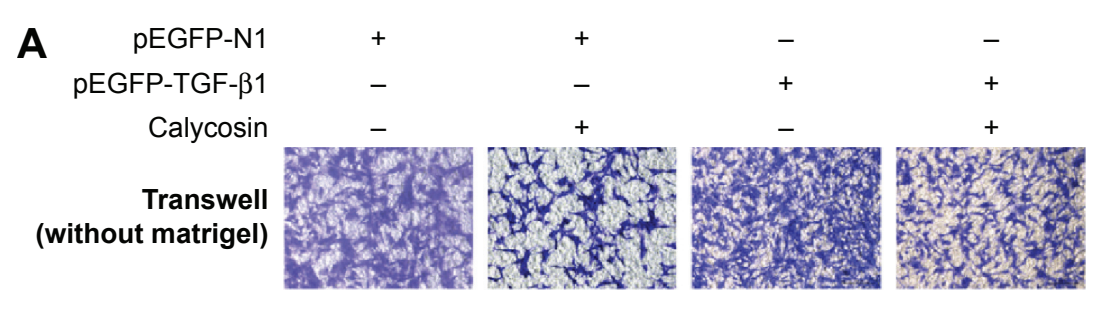

B
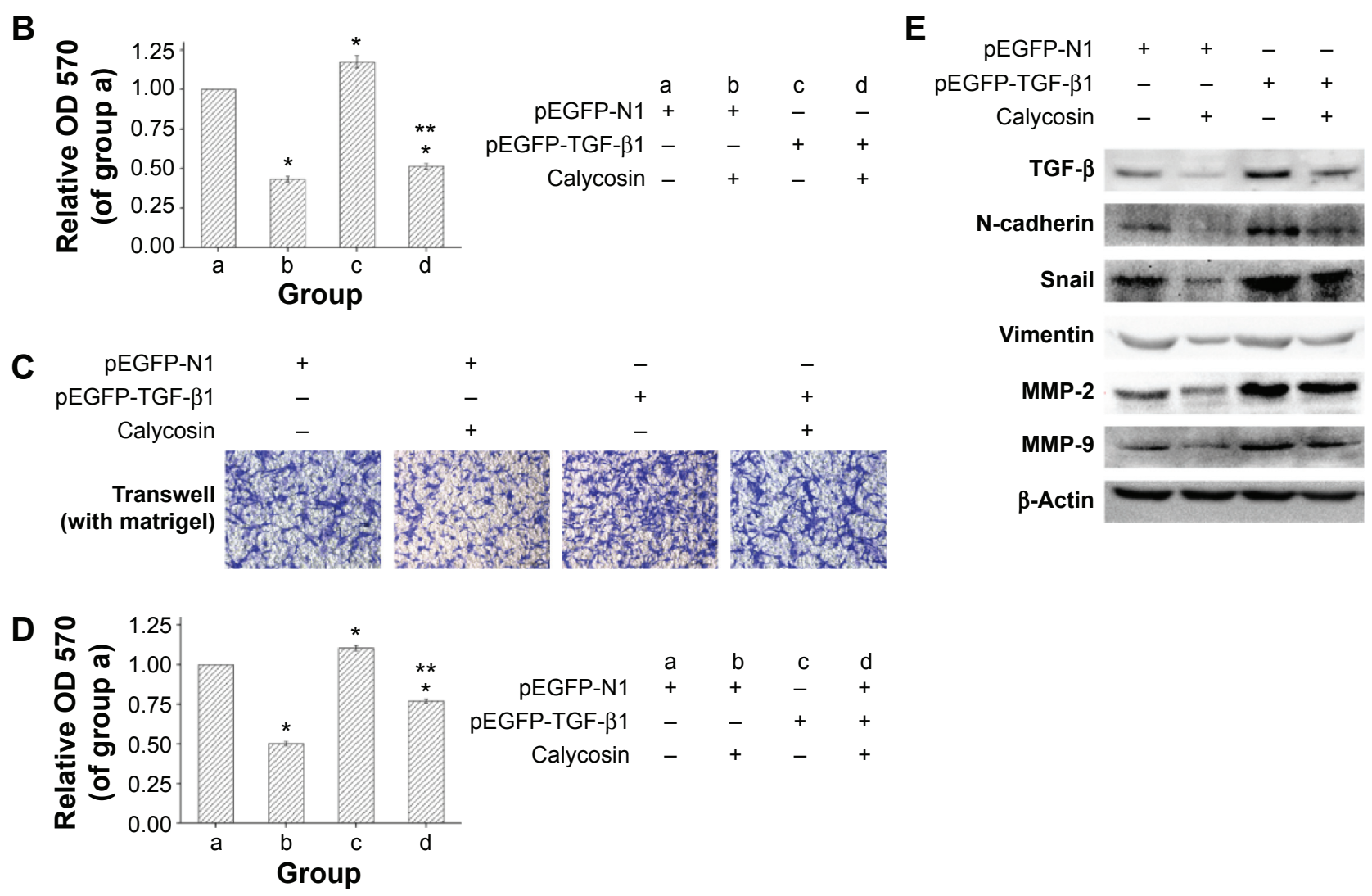

Figure 5 Overexpression of transforming growth factor beta (TGF- $\beta$ ) reduced response to calycosin.

Notes: U87 were transfected with TGF- $\beta$ I expression plasmid or empty vector with or without calycosin. (A and B) Twenty-four hours after the transfection, cells were treated with $200 \mu \mathrm{M}$ calycosin or vehicle for another 18 hours. The representative images of migrate cells at the bottom of the membrane stained with crystal violet were visualized; the stained cells were eluted by $33 \%$ glacial acetic acid for 20 minutes. Relative OD 570 was obtained and normalized to that of group a. (C and $\mathbf{D}$ ) Transfected cells were incubated with calycosin for 24 hours. Photographs of the cell invasion through the polycarbonate membrane stained by crystal violet, relative OD 570 were obtained and normalized to that of group a. (E) Overexpression of TGF- $\beta$ restored the protein level of itself and downstream molecules. Twenty-four hours after transfection, cells were incubated with calycosin or vehicle for another 24 hours. Cell extracts from each group were immunoblotted for TGF- $\beta$, N-cadherin, Snail, Vimentin, MMP-2, and MMP-9, respectively. $* P<0.05$, compared with group a. $* * P<0.05$ compared with group $b$. The data are representative of three independent experiments and are presented as the mean \pm standard deviation. + Means plasmid transfected or calycosin was added; - means plasmid was not transfected or calycosin was not added (vehicle was added).

Abbreviations: MMP, matrix metalloproteinase; OD, optical density.

\section{Discussion}

Herbals are valuable sources of medicinally important substances. However, they are mixtures of chemicals with different or even opposite functions. Thus, the purified key compounds from herbals are more suitable as therapeutic drugs for further investigation. Calycosin is a bioactive chemical derived from Radix astragali. It has been extensively studied for its proangiogenesis, anti-inflammatory, and neuroprotective effects. ${ }^{15}$ Recently, the involvement of its antitumor activities was proposed. ${ }^{12,13}$ According to these studies, calycosin was able to suppress cancer cell lines by inducting apoptosis or inhibiting proliferation. However, its underlying mechanism has not been fully investigated. Moreover, the therapeutic potential of this isoflavonoid on human glioblastomas has not been evaluated either.

Excessive migration and invasion are hallmarks of malignant tumors. Genetic controls and biochemical mechanisms underlying the acquisition of migrative and invasive phenotypes and the subsequent systemic spread of cancer cells have been areas of intensive research. Approaches that specifically inhibit or reverse these malignant features have 
A

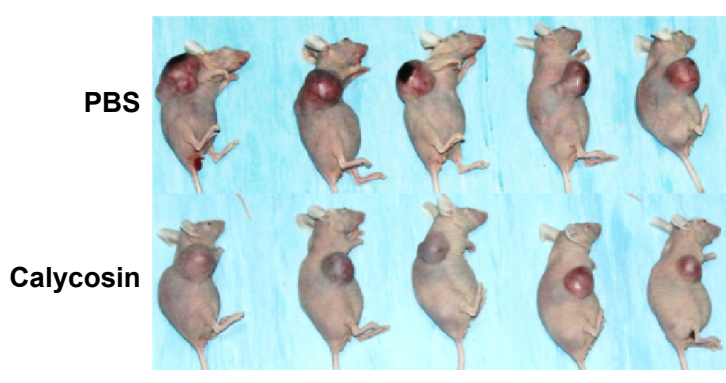

C

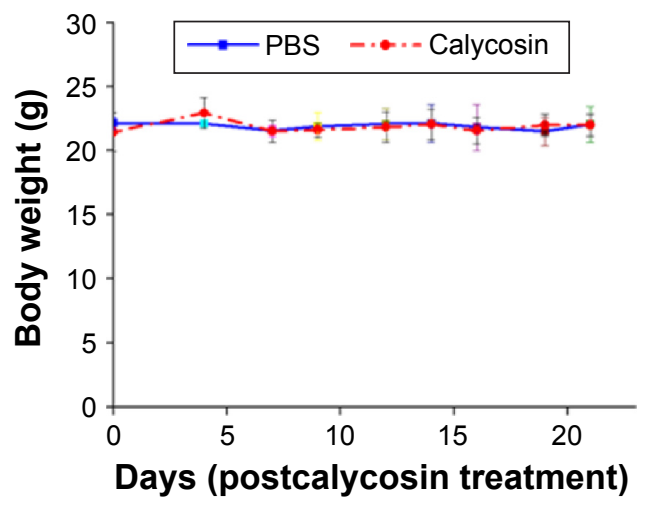

B

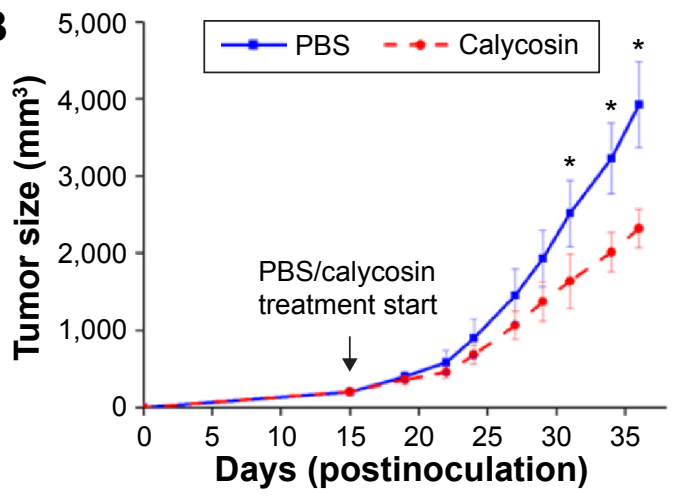

D

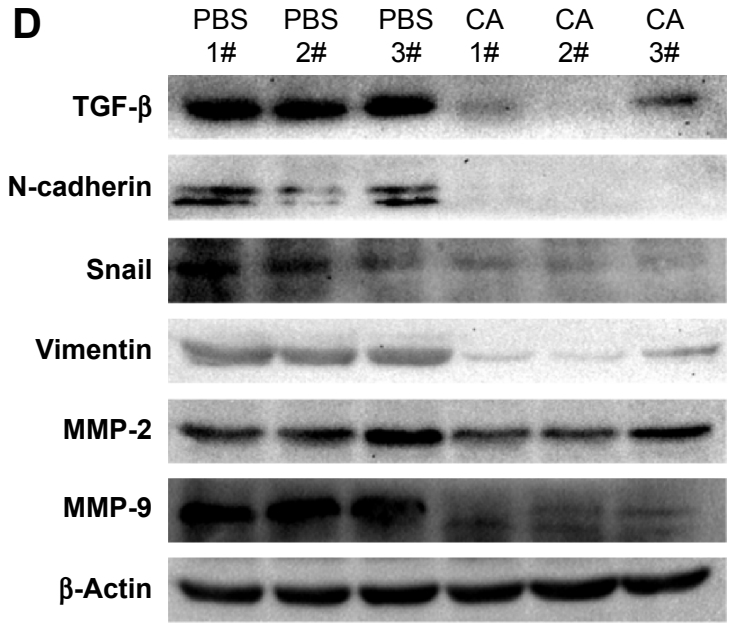

Figure 6 In vivo antitumor effects of calycosin in a xenograft model.

Notes: Mice with U87 xenografts were injected every other day with $7.5 \mathrm{mg} / \mathrm{kg}$ calycosin or PBS. (A) Representative photographs of the gross U87 tumors from nude mice treated with calycosin or PBS. (B and C) The tumor volume and body weight were measured two to three times per week. (D) At the end of the experiment, tumor tissues were excised from mice, and the protein lysates ( $40 \mu \mathrm{g}$ per lane) extracted from the tumor tissues were assessed by immunoblotting for TGF- $\beta$, N-cadherin, Snail, Vimentin, MMP-2, MMP-9, and $\beta$-actin, respectively. $* P<0.05$ compared with the PBS group.

Abbreviations: $C A$, calycosin; MMP, matrix metalloproteinase; PBS, phosphate-buffered saline.

been demonstrated valuable in some basic and preclinical studies. ${ }^{16,17}$ As shown in Figure 2, the antimigration and anti-invasion potential of calycosin in a dose-dependent manner was verified, which showed therapeutic potential of this flavonoid in glioblastoma.

EMT is a biological process that allows immobile epithelial cells to undergo alterations leading to the acquisition of a mesenchymal phenotype. In many studies, activation of an EMT program has been proposed as the critical mechanism for the acquisition of malignant phenotypes in epithelial cancer cells..$^{18,19}$ Newly discovered anticancer reagents such as AC1MMYR2, genistein, and resveratrol particularly inhibited EMT process showing antimigration or anti-invasion properties in various cancer cell types. ${ }^{4,20,21}$ Unlike in epithelial cancer cells, this malignant process has not been widely accepted in glioblastomas for its neuroepithelial origin. However, there were evidences demonstrating that primary glioblastoma and its stem cell lines express cellular and molecular characteristics of mesenchymal stem cells. ${ }^{16}$ Previous investigation also found that glioblastoma patients who responded to therapy and had longer time before recurrence had lower levels of the mesenchymal signatures in their tumor samples. ${ }^{17}$ In addition, overexpression of molecules associated with mesenchymal properties or activator of this process such as ZEB2, SNAI2/Slug, and TWIST significantly promoted migration, invasion, and progression in glioblastomas. ${ }^{22-24}$ Thus, it indicated that EMT or mesenchymal (properties) might be an ideal target for cancer therapy not only in epithelial cancer cells, but also in glioblastomas.

MMPs are a family of zinc-dependent endopeptidases that are required for extracellular matrix degradation. ${ }^{25,26}$ Among all MMPs, MMP-2 and MMP-9 play important roles for basement membrane type IV collagen degradation during cancer progression, especially for promoting tumor migration and invasion..$^{27,28}$ The two MMPs were all expressed in 
human glioblastomas; real-time polymerase chain reaction and gelatin zymography showed that both of them in glioblastoma specimens were higher than in normal tissues. In particular, MMP-9 level was strongly correlated with tumor grade. ${ }^{29}$ Similar studies performed later also indicated that MMP-2 was associated with tumor invasion, angiogenesis, metastasis, and recurrence. ${ }^{30,31}$ Moreover, experiments successfully inhibited or delayed cancer invasion and migration via modulation of MMP-2 and MMP-9. ${ }^{32-35}$ Besides the direct regulation of basement membrane degradation, there is a cross talk between MMPs and EMT that can regulate the invasion or migration in cancer cells: overexpression of MMP-2 or MMP-9 led to induction of EMT in breast cancer cells, ${ }^{36,37}$ while other investigations revealed that a variety of EMT processes could induce MMPs' expression, such as Snail, a well-known EMT activator, which also proved to be an inducer of MMP-2 expression, suggesting that EMT contributes to the increased invasion not only through inhibition of cell-cell adhesion but also via upregulation of MMP-2 expression in cancer cells. ${ }^{38,39}$ Thus, as mentioned earlier, the positive feedback loop between MMPs and EMT synergistically contributes to the excessive migration and invasion in malignant tumors.

Therefore, strategies inhibiting both mesenchymal properties and MMPs may be an efficient way for cancer therapy. As shown in Figure 3, downregulation of mesenchymal markers/ activators such as N-cadherin, Snail, and Vimentin indicated the inhibitory effects of calycosin on cell mesenchymal properties. On the other hand, MMP-2 and MMP-9 were also significantly reduced after calycosin treatment. Taken together, solid evidences proved that calycosin inhibited not only mesenchymal properties but also MMPs in U87 and U251 in vitro. These effects may contribute to its antimigration and anti-invasion potential in human glioblastoma cells.

We found that U87 was more sensitive to calycosin, although it showed more malignancy in proliferation, migration, and invasion than U251. Different from U87, U251 cells have $p 53$-null status and $p 53$ gene not only participates in cell proliferation and apoptosis but also influences EMT process and its related migration and invasion. ${ }^{40-43}$ Therefore, we presume that U87 is more sensitive to U251 probably because of $p 53$ mutation in U251 cell line, which makes it resistant to calycosin-induced proliferation inhibition and other effects. Further experiments on this subject should be done to verify this hypothesis.

TGF- $\beta$ is secreted as an inactive form in which the mature growth factor remains associated with its propeptide.
To elicit a biological response, TGF- $\beta$ is released, active TGF- $\beta$ binds to TGF- $\beta$ receptors (TGF- $\beta$ RI and TGF- $\beta$ RII), and initiates signal transduction. Accumulated evidence suggested that TGF- $\beta$ plays a regulatory role in the EMT process and MMP activation in various cancer cells. ${ }^{40-43}$ Based on these findings, we further studied the expression level of TGF- $\beta$ in both cell lines. Figure 4 clearly showed that both mRNA and protein levels of this molecule were significantly attenuated after calycosin treatment. So we wonder whether TGF- $\beta$ was a critical target of calycosin. As expected, forced expression of TGF- $\beta 1$ in calycosin-treated U87 cells prevented its mesenchymal properties as well as MMP-2 and MMP-9 expression in comparison with their respective control groups (Figure 5). It suggests that calycosin might target, at least in part, TGF- $\beta$ in the glioblastoma cells. This means further investigations are needed to clarify the direct binding target of calycosin. Although a previous study proved that phenol like spectomycin B1 could directly bind to ubiquitin ligase and induce target protein degradation, ${ }^{44} \mathrm{a}$ few other phenols may directly interfere with gene promoter to regulate its expression. ${ }^{45}$ The direct target of calycosin has not been reported and researches on other phenols can hardly provide guidance since these direct targets of phenols did not stick to some pattern. Therefore, rigorous bioinformatics and high-throughput screening might be the best way to find the direct target of calycosin.

To extend the in vitro observations, in vivo experiments were performed. Our results demonstrated that the application of intravenous calycosin could be efficiently delivered in vivo, significantly suppressed the growth of established glioblastoma xenografts, and caused no loss in body weights (Figure 6A and B). It provided calycosin as a potential anticancer drug and relatively low toxicity to normal tissues. More importantly, the molecules such as TGF- $\beta$, N-cadherin, Snail, Vimentin, MMP-2, and MMP-9 in tumor tissues were all downregulated after calycosin treatment, which was consistent with in vitro findings (Figure 6C).

\section{Conclusion}

In this current study, we present the first report of anticancer activity of calycosin in glioblastoma cells in vitro and vivo. This is also the first evidence that calycosin acts as a suppresser of cell migration and invasion. Calycosin-induced downregulation of TGF- $\beta$ leads to loss of mesenchymal properties and inactivation of MMP-2 and MMP-9 was proved to be involved in this process. Collectively, we thus conclude calycosin is a promising regimen to treat glioblastoma. 


\section{Acknowledgment}

This work was supported by grant from the "National Natural Science Foundation of China” (Grant No 81573774).

\section{Disclosure}

The authors report no conflicts of interest in this work.

\section{References}

1. Jansen M, Yip S, Louis DN. Molecular pathology in adult gliomas: diagnostic, prognostic, and predictive markers. Lancet Neurol. 2010; 9(7):717-726.

2. Stummer W, Meinel T, Ewelt C, et al. Prospective cohort study of radiotherapy with concomitant and adjuvant temozolomide chemotherapy for glioblastoma patients with no or minimal residual enhancing tumor load after surgery. $J$ Neurooncol. 2012;108(1):89-97.

3. Ellingson BM, Cloughesy TF, Zaw T, et al. Functional diffusion maps (fDMs) evaluated before and after radiochemotherapy predict progression-free and overall survival in newly diagnosed glioblastoma. Neuro Oncol. 2012;14(3):333-343.

4. Zhang LL, Li L, Wu DP, et al. A novel anti-cancer effect of genistein: reversal of epithelial mesenchymal transition in prostate cancer cells. Acta Pharmacol Sin. 2008;29(9):1060-1068.

5. Kalluri R, Weinberg RA. The basics of epithelial-mesenchymal transition. J Clin Invest. 2009;119(6):1420-1428.

6. Han SP, Kim JH, Han ME, et al. SNAI1 is involved in the proliferation and migration of glioblastoma cells. Cell Mol Neurobiol. 2011;31(3): 489-496.

7. Mikheeva SA, Mikheev AM, Petit A, et al. TWIST1 promotes invasion through mesenchymal change in human glioblastoma. Mol Cancer. 2010; 9:194.

8. Phillips HS, Kharbanda S, Chen R, et al. Molecular subclasses of highgrade glioma predict prognosis, delineate a pattern of disease progression, and resemble stages in neurogenesis. Cancer Cell. 2006;9(3): $157-173$.

9. Xu Y, Feng L, Wang S, et al. Calycosin protects HUVECs from advanced glycation end products-induced macrophage infiltration. J Ethnopharmacol. 2011;137(1):359-370.

10. Guo C, Tong L, Xi M, Yang H, Dong H, Wen A. Neuroprotective effect of calycosin on cerebral ischemia and reperfusion injury in rats. J Ethnopharmacol. 2012;144(3):768-774.

11. Zhu H, Zhang Y, Ye G, Li Z, Zhou P, Huang C. In vivo and in vitro antiviral activities of calycosin-7-O-beta-D-glucopyranoside against coxsackie virus B3. Biol Pharm Bull. 2009;32(1):68-73.

12. Qiu R, Ma G, Zheng C, et al. Antineoplastic effect of calycosin on osteosarcoma through inducing apoptosis showing in vitro and in vivo investigations. Exp Mol Pathol. 2014;97(1):17-22.

13. Chen J, Zhao X, Ye Y, Wang Y, Tian J. Estrogen receptor betamediated proliferative inhibition and apoptosis in human breast cancer by calycosin and formononetin. Cell Physiol Biochem. 2013;32(6): 1790-1797.

14. Chen J, Hou R, Zhang X, Ye Y, Wang Y, Tian J. Calycosin suppresses breast cancer cell growth via ERbeta-dependent regulation of IGF-1R, p38 MAPK and PI3K/Akt pathways. PloS One. 2014;9(3): e91245.

15. Gao J, Liu ZJ, Chen T, Zhao D. Pharmaceutical properties of calycosin, the major bioactive isoflavonoid in the dry root extract of Radix astragali. Pharm Biol. 2014;52(9):1217-1222.

16. Qin G, Xu F, Qin T, et al. Palbociclib inhibits epithelial-mesenchymal transition and metastasis in breast cancer via c-Jun/COX-2 signaling pathway. Oncotarget. 2015;6(39):41794-41808.

17. Taurin S, Nimick M, Larsen L, Rosengren RJ. A novel curcumin derivative increases the cytotoxicity of raloxifene in estrogen receptornegative breast cancer cell lines. Int J Oncol. Epub 2015 Nov 19.
18. Kwak TW, Shin HJ, Jeong YI, et al. Anticancer activity of streptochlorin, a novel antineoplastic agent, in cholangiocarcinoma. Drug Des Devel Ther. 2015;9:2201-2214.

19. Abu N, Mohamed NE, Yeap SK, et al. In vivo antitumor and antimetastatic effects of flavokawain B in 4T1 breast cancer cell-challenged mice. Drug Des Devel Ther. 2015;9:1401-1417.

20. Shi Z, Zhang J, Qian X, et al. AC1MMYR2, an inhibitor of dicer-mediated biogenesis of Oncomir miR-21, reverses epithelialmesenchymal transition and suppresses tumor growth and progression. Cancer Res. 2013;73(17):5519-5531.

21. Wang H, Zhang H, Tang L, et al. Resveratrol inhibits TGF-beta1induced epithelial-to-mesenchymal transition and suppresses lung cancer invasion and metastasis. Toxicology. 2013;303:139-146.

22. Elias MC, Tozer KR, Silber JR, et al. TWIST is expressed in human gliomas and promotes invasion. Neoplasia. 2005;7(9):824-837.

23. Yang HW, Menon LG, Black PM, Carroll RS, Johnson MD. SNAI2/ Slug promotes growth and invasion in human gliomas. BMC Cancer. 2010;10:301.

24. Qi S, Song Y, Peng Y, et al. ZEB2 mediates multiple pathways regulating cell proliferation, migration, invasion, and apoptosis in glioma. PloS One. 2012;7(6):e38842.

25. Kessenbrock K, Wang CY, Werb Z. Matrix metalloproteinases in stem cell regulation and cancer. Matrix Biol. 2015;44-46:184-190.

26. Itoh Y. Membrane-type matrix metalloproteinases: their functions and regulations. Matrix Biol. 2015;44-46:207-223.

27. Schmalfeldt B, Prechtel D, Harting K, et al. Increased expression of matrix metalloproteinases (MMP)-2, MMP-9, and the urokinase-type plasminogen activator is associated with progression from benign to advanced ovarian cancer. Clin Cancer Res. 2001;7(8):2396-2404.

28. Zeng ZS, Cohen AM, Guillem JG. Loss of basement membrane type IV collagen is associated with increased expression of metalloproteinases 2 and 9 (MMP-2 and MMP-9) during human colorectal tumorigenesis. Carcinogenesis. 1999;20(5):749-755.

29. Forsyth PA, Wong H, Laing TD, et al. Gelatinase-A (MMP-2), gelatinase-B (MMP-9) and membrane type matrix metalloproteinase-1 (MT1-MMP) are involved in different aspects of the pathophysiology of malignant gliomas. Br J Cancer. 1999;79(11-12):1828-1835.

30. Komatsu K, Nakanishi Y, Nemoto N, Hori T, Sawada T, Kobayashi M. Expression and quantitative analysis of matrix metalloproteinase-2 and -9 in human gliomas. Brain Tumor Pathol. 2004;21(3):105-112.

31. Wang M, Wang T, Liu S, Yoshida D, Teramoto A. The expression of matrix metalloproteinase-2 and -9 in human gliomas of different pathological grades. Brain Tumor Pathol. 2003;20(2):65-72.

32. Lin SS, Lai KC, Hsu SC, et al. Curcumin inhibits the migration and invasion of human A549 lung cancer cells through the inhibition of matrix metalloproteinase-2 and -9 and vascular endothelial growth factor (VEGF). Cancer Lett. 2009;285(2):127-133.

33. Deshane J, Garner CC, Sontheimer H. Chlorotoxin inhibits glioma cell invasion via matrix metalloproteinase-2. J Biol Chem. 2003;278(6): 4135-4144.

34. Kargiotis O, Chetty C, Gondi CS, et al. Adenovirus-mediated transfer of siRNA against MMP-2 mRNA results in impaired invasion and tumor-induced angiogenesis, induces apoptosis in vitro and inhibits tumor growth in vivo in glioblastoma. Oncogene. 2008;27(35): 4830-4840.

35. Chen Y, Zheng L, Liu J, et al. Shikonin inhibits prostate cancer cells metastasis by reducing matrix metalloproteinase-2/-9 expression via $\mathrm{AKT} / \mathrm{mTOR}$ and ROS/ERK1/2 pathways. Int Immunopharmacol. 2014;21(2):447-455.

36. Kim ES, Sohn YW, Moon A. TGF-beta-induced transcriptional activation of MMP-2 is mediated by activating transcription factor (ATF)2 in human breast epithelial cells. Cancer Lett. 2007;252(1): $147-156$.

37. Kim IY, Yong HY, Kang KW, Moon A. Overexpression of ErbB2 induces invasion of MCF10A human breast epithelial cells via MMP-9. Cancer Lett. 2009;275(2):227-233. 
38. Miyoshi A, Kitajima Y, Kido S, et al. Snail accelerates cancer invasion by upregulating MMP expression and is associated with poor prognosis of hepatocellular carcinoma. Br J Cancer. 2005;92(2):252-258.

39. Yokoyama K, Kamata N, Fujimoto R, et al. Increased invasion and matrix metalloproteinase- 2 expression by Snail-induced mesenchymal transition in squamous cell carcinomas. Int J Oncol. 2003;22(4): 891-898.

40. Lu WJ, Chua MS, So SK. Suppression of ATAD2 inhibits hepatocellular carcinoma progression through activation of p53- and p38-mediated apoptotic signaling. Oncotarget. 2015;6(39):41722-41735.

41. Lin Y, Mallen-St Clair J, Luo J, Sharma S, Dubinett S, St John M. p53 modulates NF-kappaB mediated epithelial-to-mesenchymal transition in head and neck squamous cell carcinoma. Oral Oncol. 2015; 51(10):921-928.
42. Jiang FZ, He YY, Wang HH, et al. Mutant p53 induces EZH2 expression and promotes epithelial-mesenchymal transition by disrupting p68Drosha complex assembly and attenuating miR-26a processing. Oncotarget. Epub 2015 Nov 18.

43. Furth N, Bossel Ben-Moshe N, Pozniak Y, et al. Down-regulation of LATS kinases alters p53 to promote cell migration. Genes Dev. 2015; 29(22):2325-2330.

44. Hirohama M, Kumar A, Fukuda I, et al. Spectomycin B1 as a novel SUMOylation inhibitor that directly binds to SUMO E2. ACS Chem Biol. 2013;8(12):2635-2642.

45. Mahmoud AM, Al-Alem U, Ali MM, Bosland MC. Genistein increases estrogen receptor beta expression in prostate cancer via reducing its promoter methylation. J Steroid Biochem Mol Biol. 2015;152:62-75. 


\section{Supplementary material}

Table SI Primers for quantitative real-time RT-PCR analysis of gene transcript expression

\begin{tabular}{llll}
\hline Gene & Forward primer & Reverse primer & Size (bp) \\
\hline TGF- $\beta$ I & CCCAGCATCTGCAAAGCTC & GTCAATGTACAGCTGCCGCA & I0I \\
$\beta$-Actin & AGCGCGGCTACAGCTTCA & GGCCATCTCTTGCTCGAAGT & I0I \\
\hline
\end{tabular}

Abbreviations: TGF- $\beta$ I, transforming growth factor beta I; RT-PCR, reverse transcription polymerase chain reaction.

\section{Publish your work in this journal}

Drug Design, Development and Therapy is an international, peerreviewed open-access journal that spans the spectrum of drug design and development through to clinical applications. Clinical outcomes, patient safety, and programs for the development and effective, safe, and sustained use of medicines are a feature of the journal, which has also been accepted for indexing on PubMed Central. The manuscript management system is completely online and includes a very quick and fair peer-review system, which is all easy to use. Visit http://www.dovepress.com/testimonials.php to read real quotes from published authors.

\footnotetext{
Submit your manuscript here: http://www.dovepress.com/drug-design-development-and-therapy-journal
} 